Diabetologia (1987) 30: 239-243

\title{
Reduced anterograde and retrograde accumulation of axonally transported phosphof ructokinase in streptozotocin-diabetic rats: effects of insulin and the aldose reductase inhibitor 'Statil'
}

\author{
G. B. Willars, N.A.Calcutt and D. R. Tomlinson \\ Department of Physiology and Pharmacology, Medical School, Queen's Medical Centre, Nottingham, UK
}

\begin{abstract}
Summary. This study examined anterograde and retrograde accumulation of axonally transported 6-phosphofructokinase activity, proximal and distal to sciatic nerve constrictions, in rats with streptozotocin-induced diabetes of 4 weeks' duration. There were deficits in accumulation on both sides of the constriction in untreated diabetic rats (proximal accumulation $66 \%$ of controls, $p<0.05$; distal accumulation $32 \%$ of controls, $p<0.01$ ). There was also a reduction in the phosphofructokinase activity per unit length unconstricted sciatic nerve in the untreated diabetic rats $(87 \%$ of controls, $p<$ 0.05 ). Treatment of an age-matched group of diabetic rats with twice-daily insulin prevented all the above changes. There were significant increases, over untreated diabetic rats, in phosphofructokinase activity accumulated at constrictions ( $p<0.01$ for both proximal and distal) and in unconstricted nerve $(p<0.05)$. Indeed the activities measured in insulintreated diabetic rats were virtually identical to those of controls. Treatment of a third group of diabetic rats with the al-
\end{abstract}

dose reductase inhibitor 'Statil' prevented or attenuated accumulations of polyol pathway metabolites and prevented depletion of myo-inositol in the sciatic nerve. In spite of these indications of effective aldose reductase inhibition, the drug was without effect on the deficits in accumulation of activity at ligatures or unconstricted nerve levels of phosphofructokinase activity. We conclude that short-term experimental diabetes in rats induced defects in both anterograde and retrograde axonal transport of 6-phosphofructokinase activity. These defects were prevented by intensive insulin treatment but were resistant to an effective aldose reductase inhibitor, indicating a lack of involvement of polyol pathway flux in their pathogenesis.

Key words: Aldose reductase, axonal flow, diabetic neuropathies, insulin, phosphofructokinase, sorbitol, streptozotocindiabetes.
Anterograde axonal transport of macromolecules and organelles synthesized in the perinuclear region of the cell body is required to maintain the structural and functional integrity of the neurone. Retrograde axonal transport of the overflow of these materials may serve as a feedback mechanism regulating perikaryl protein synthesis [1]. The vulnerability of the neurone to disorders of axonal transport is shown by the failure of synaptic transmission and degeneration of nerve terminals occurring after the experimental impairment of transport processes [2,3]. Defective axonal transport might therefore contribute to the peripheral axonopathy characteristic of symmetrical polyneuropathies in patients with chronic diabetes mellitus.

The majority of the studies on axonal transport in experimental diabetes have been reviewed elsewhere [4]. Slow anterograde transport of axoplasmic proteins has shown the most reproducible defects. Of particular interest are deficits in velocity and amount of radio- labelled proteins travelling at $1-2 \mathrm{~mm} /$ day [5-7] and reduced amount of the enzyme choline acetyltransferase $[8,9]$. Both of these defects are prevented in rats with short-term diabetes by 'tight' control of blood glucose with insulin [5-10]. In contrast, the former defect is resistant to inhibition of aldose reductase or myo-inositol treatment $[6,7]$, whilst the latter can be prevented or reversed by either sorbinil or 'Statil' [8,9], as well as by treatment with myo-inositol [8].

We have recently demonstrated anterograde and retrograde axonal transport of 6-phosphofructokinase (EC 2.7.1.11) activity by measuring its accumulation both proximal and distal to a constricting ligature applied to the mid-femoral region of the sciatic nerve in the rat. The accumulations on both sides of the ligature were linear for up to $24 \mathrm{~h}$ [11]. In order to extend observations of axonal transport in diabetes to novel components of axoplasm, particularly to those elements not directly related to neurotransmitters and 
their metabolism, we studied the accumulation of phosphofructokinase proximal and distal to sciatic nerve ligations in rats with short-term streptozotocindiabetes. The effects of insulin and aldose reductase inhibitor treatment were examined. Study of this enzyme may have broader implications due to its key role in the regulation of glycolysis [12] and the resistance to ischaemic conduction block in diabetic man $[13,14]$ and animals $[15,16]$, which may be due, at least in part, to an increased ATP production by anaerobic glycolysis [16].

\section{Materials and methods}

\section{Experimental organisation and treatment regimes}

Male Wistar rats (age 15-16 weeks; weight range 290-320 g) were assigned at random to four groups and fasted overnight. The following morning three groups were made diabetic by a single intra-peritoneal injection of $50 \mathrm{mg} / \mathrm{kg}$ streptozotocin (ICI Pharmaceuticals Division, Alderley Park, Macclesfield, UK) dissolved immediately prior to injection in sterile saline $(154 \mathrm{mmol} / \mathrm{NaCl})$. The remaining group of rats served as controls and were injected with saline only. Three days later blood samples were collected by tail prick from the streptozotocin-injected rats and blood glucose concentration determined by the use of a glucose oxidase strip-operated reflectance meter ('Reflomat'; Boehringer Corporation, London, UK). Animals with blood glucose concentrations of less than $15 \mathrm{mmol} / 1$ were rejected from the study. Immediately following streptozotocin treatment the rats of one group were placed on a diet (41B (Modified); Heygate \& Sons, Northampton, UK) containing the aldose reductase inhibitor 'Statil' (ICI 128436; 3-(4-bromo-2-fluorobenzyl)-4-oxo-3Hphthalazin-1-ylacetic acid) at $400 \mathrm{mg} / \mathrm{kg}$ diet. Of the two remaining diabetic groups, one was untreated while the other received insulin to control the diabetes. Three days following streptozotocin injection each rat designated for insulin treatment was given a starting dose of $2.5 \mathrm{U} / 100 \mathrm{~g}$ Semitard insulin MC (Novo Industri A/S, Copenhagen, Denmark) by subcutaneous injection on the morning of blood glucose measurement and 2.5 units $/ 100 \mathrm{~g}$ Monotard insulin MC (Novo Industri $A / S$ ) on the evening of that day. Thereafter blood glucose was measured each morning; animals with blood glucose values of $>9 \mathrm{mmol} / 1$ were given a morning dose of 2 units $/ 100 \mathrm{~g}$ Semitard insulin and an evening dose of 2 units $/ 100 \mathrm{~g}$ Monotard insulin. Animals with a morning blood glucose value of $<9 \mathrm{mmol}$ were not injected with Semitard insulin that morning but were given an evening dose of 2 units $/ 100 \mathrm{~g}$ Monotard insulin provided that their blood glucose level had risen to $>9 \mathrm{mmol} / 1$.

After 4 days insulin treatment, the daytime response was examined in detail by taking blood for glucose estimation (reflectance meter) at hourly intervals from 09.30 to 16.30 hours. The mean blood glucose for the whole group over this period was $6.6 \pm 1.2 \mathrm{mmol} / 1$. Four days later, when the rats' tails had healed, the nocturnal blood glucose was examined in detail by taking two-hourly measurements from 17.00 to 10.00 hours the next day. The mean group value was $10.2 \pm 1.8 \mathrm{mmol} / \mathrm{l}$. On this basis the treatment was adjudged to be satisfactory.

From Monday evening to Friday morning of weeks 2, 3 and 4 the daily consumption of food and water was measured for each box of rats. Values were estimated per rat; no statistical analysis was possible.

\section{Axonal transport studies}

Four weeks following streptozotocin injection, under brief recovery halothane anaesthesia, a single ligation was tied tightly around the mid-femoral portion of the left sciatic nerve. Insulin-treated animals were not injected on the morning of operation, but were given $1 \mathrm{U} /$ $100 \mathrm{~g}$ Monotard insulin in the evening provided blood glucose values were $>9 \mathrm{mmol} / 1$. Animals were killed $24 \mathrm{~h}$ later by a blow to the head and bled from the throat. A blood sample was taken at the time of death, centrifuged $(9000 \mathrm{~g}, 1 \mathrm{~min})$ and the plasma glucose determined by a hydrogen peroxidase-sensitive static phase glucose oxidase-operated glucose analyser (Model 23AM; Yellow Springs Instrument Co., Yellow Springs, Ohio, USA). Both sciatic nerves were removed and frozen under moderate tension on a brass block cooled in liquid nitrogen. Nerves were cut into $3 \mathrm{~mm}$ segments; 2 proximal and 1 distal to the ligation. The equivalent region of the contralateral (unligated) nerve was treated similarly and cut to give three $3 \mathrm{~mm}$ segments to permit determination of background activity. Segments were stored at $-80^{\circ} \mathrm{C}(<1$ week) until assay of phosphofructokinase activity.

\section{Assay of phosphofructokinase activity}

Nerve segments were homogenized in glass-in-glass hand homogenizers in $0.2 \mathrm{ml}$ of ice-cold extraction buffer consisting of $50 \mathrm{mmol} / 1$ Tris, $1 \mathrm{mmol} / 1$ EDTA, $5 \mathrm{mmol} / 1 \mathrm{MgCl}_{2}$ and $1 \%$ Triton $\mathrm{x}-100$ at $\mathrm{pH}$ 8.2. Phosphofructokinase activity was determined immediately, in the supernatant of centrifuged $(9000 \mathrm{~g}, 1 \mathrm{~min})$ homogenates, spectrophotometrically at $25^{\circ} \mathrm{C}$ on a Gilford recording spectrophotometer (Model 240) by monitoring the oxidation of $\mathrm{NADH}_{2}$ at $340 \mathrm{~nm}$ using the method of Opie and Newsholme [17]. Reaction cuvettes contained in a volume of $1 \mathrm{ml}$ at $\mathrm{pH} 8.2: 50 \mathrm{mmol} / 1 \mathrm{Tris}, 6 \mathrm{mmol} / 1$ $\mathrm{MgCl}_{2}, 200 \mathrm{mmol} / \mathrm{l} \mathrm{KCI}, 1 \mathrm{mmol} / 1$ mercaptoethanol, $2 \mathrm{mmol} / 1$ AMP, $1 \mathrm{mmol} / 1$ ATP, $0.16 \mathrm{mmol} / 1 \mathrm{NADH}_{2}, 0.09 \mathrm{mmol} / 1$ antimycin A, 3 mmol/1 fructose-6-phosphate, $45.5 \mu \mathrm{g}$ glycerol-3-phosphate dehydrogenase, $4.5 \mu \mathrm{g}$ triosephosphate isomerase and $50 \mu \mathrm{g}$ aldolase. Reactions were started by the addition of substrate (fructose 6-phosphate) and followed in duplicate for $10 \mathrm{~min}$. Cuvettes containing no substrate were run as blanks for each nerve segment and generally did not show any detectable oxidation of $\mathrm{NADH}_{2}$. Enzyme activity was calculated as nmol of substrate utilized per min per $3 \mathrm{~mm}$ segment of nerve. All chemicals for this assay were obtained from Boehringer Mannheim Biochemica, Lewes, East Sussex, UK.

\section{Calculation of phosphofructokinase accumulation}

Background phosphofructokinase activity was calculated as the mean activity in the three segments from the unconstricted nerve. Our previous study [11] showed that proximal accumulation at 24-h sciatic nerve ligatures is confined to the $6 \mathrm{~mm}$ nerve immediately adjacent to the site of crush. Distal accumulation is restricted to the $3 \mathrm{~mm}$ nerve on the other side of the ligature. Accordingly, net anterograde accumulation was calculated as the activity in the two segments proximal to the ligature minus twice the background activity and net retrograde accumulation as the activity in the segment distal to the ligation minus the background activity.

\section{Determination of nerve sugars and polyols}

The remaining portion of the unconstricted contralateral nerve was used for determination of nerve sugar and polyol levels by gas liquid chromatography of their extracted trimethylsilyl derivatives using $\alpha$ methyl mannoside as an internal standard [9].

\section{Statistical analysis}

For each variable (except food and water intake) data were subjected to a one-way analysis of variance (ANOVA) using the Statistical Package for Social Scientists (SPSS-X Incorporated, Chicago, Ill, USA) run on an ICL 2900 computer. Where the $\mathrm{F}$ ratio gave $p<$ 0.05 , comparisons between individual group means were made by Duncan's multiple range test [18] at significance levels of 0.05 and 0.01 . Data are presented as mean $\pm \mathrm{SD}$ except where stated other- 
Table 1. Axonal transport data and background (unconstricted nerve) levels of 6-phosphofructokinase (PFK) activity and nerve sugar and polyol levels for controls and for groups of streptozotocin-diabetic (duration 4 weeks) rats. Diabetic rats were untreated, treated daily with the aldose reductase inhibitor 'Statil' (ICI 128436) or treated twice daily with insulin

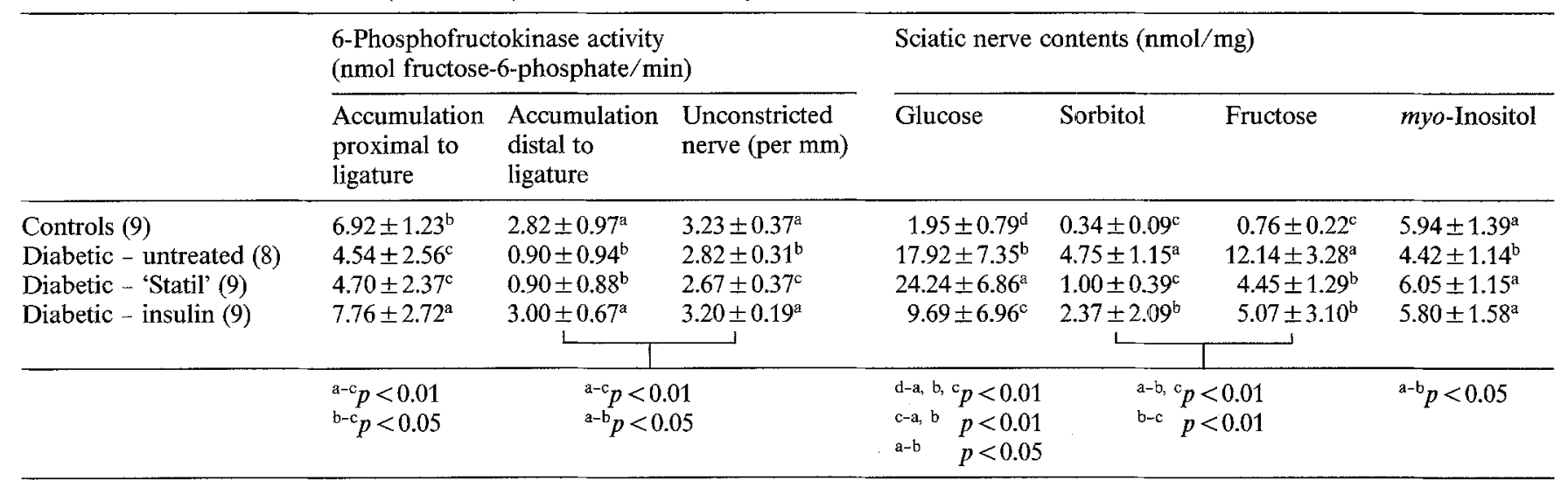

Data are mean $\pm S D$; numbers of rats in brackets. For explanation of superscript codes for significance of differences see Materials and Methods. Proximal accumulation of PFK activity was calculated by subtraction of the activity in $6 \mathrm{~mm}$ unconstricted nerve from that in $6 \mathrm{~mm}$ nerve proximal to the ligature. Distal accumulation was calculated by subtraction of the activity in $3 \mathrm{~mm}$ unconstricted nerve from that in $3 \mathrm{~mm}$ nerve distal to the ligature

wise. Numbers of rats per group are given in Table 1 . The use of Duncan's range test enabled multiple comparisons including any group mean. The resulting permutations for establishment of significance of difference are encoded as letter superscripts in Table 1. The codes are explained at the foot of each column. For example, in Table 1, column 4 (nerve glucose), the coding at the foot of the column indicates that the mean superscripted ' $\mathrm{d}$ ' (controls) differed significantly from all others (' $a$ ', 'b' and 'c') at the level $p<0.01$. The mean superscripted ' $c$ ' (insulin-treated diabetic) differed from the sub-sets superscripted ' $a$ ' and 'b' (untreated and 'Statil'-treated diabetic) at the level $p<0.01$. Finally the group superscripted 'a' ('Statil'-treated diabetic) differed from the group superscripted ' $b$ ' (untreated diabetic) at the level $p<0.05$.

\section{Results}

\section{Food, water, body weight and blood glucose}

The mean daily food and water intake (measured per box, but transposed to single rat dimensions) over the last 3 weeks of the protocol were $26 \mathrm{~g}$ and $37 \mathrm{ml}$ for controls, $31 \mathrm{~g}$ and $85 \mathrm{ml}$ for insulin-treated diabetic, $38 \mathrm{~g}$ and $164 \mathrm{ml}$ for untreated diabetic and $37 \mathrm{~g}$ and $157 \mathrm{ml}$ for 'Statil'-treated diabetic rats. Thus, diabetes induced marked polydipsia and hyperphagia which was attenuated by insulin, but unaffected by 'Statil'.

Body weight changes are shown in Figure 1 . The untreated and 'Statil'-treated diabetic rats lost weight progressively, with final values of $237 \pm 23 \mathrm{~g}$ and $223 \pm 24 \mathrm{~g}$ respectively. These did not differ significantly. The group which received insulin showed an initial weight loss (before treatment began) but then gained weight steadily (Fig.1), although their terminal mean value $(304 \pm 22)$ was significantly $(p<0.01)$ less than that of the control group (338 \pm 10$)$.

The untreated and 'Statil'-treated diabetic rats were markedly hyperglycaemic at death (plasma glucose

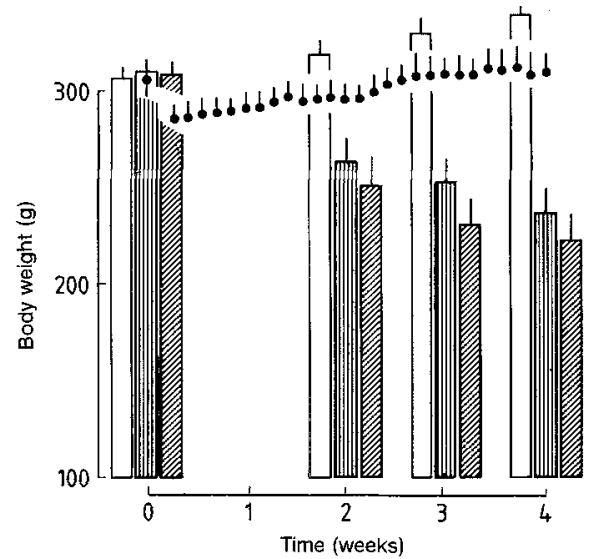

Fig. 1. Changes in body weight throughout the study. Columns show body weights of control rats ( $\square)$, untreated diabetic rats ( $\mathbb{U}$ ) and 'Statil'-treated rats ( 10 measured immediately before streptozotocin injection (day 0 ) and 2,3 and 4 weeks later. Daily body weights for the insulin-treated diabetic rats are shown as - Data are mean $\pm \mathrm{SD}$

values, $37.4 \pm 5.1$ and $39.6 \pm 7.1 \mathrm{mmol} / \mathrm{1}$, respectively). The control group had a final plasma glucose value of $6.6 \pm 0.7 \mathrm{mmol} / \mathrm{l}$. Since the insulin doses given to the other group were halved over the final $24 \mathrm{~h}$ (see Materials and methods), their final value of $22.4 \pm$ $10.9 \mathrm{mmol} / 1$ plasma glucose does not reflect accurately the efficacy of treatment.

\section{Nerve phosphofructokinase activity}

These data are presented in Table 1 . The activity of phosphofructokinase in segments of the unconstricted right sciatic nerve, used for determination of background activity, was significantly reduced in untreated diabetic rats $(p<0.05)$. This reduction was unaffected 
by 'Statil' treatment, but was totally prevented by the insulin treatment regime. Similarly, the accumulations of phosphofructokinase activity, both proximal and distal to the ligation, were significantly reduced in diabetic animals whether untreated or treated with 'Statil' $(p<0.05)$. Insulin treatment of diabetic animals prevented both defects.

\section{Nerve sugar and polyol contents}

The untreated diabetic rats had markedly elevated nerve glucose levels (Table 1 ); these were significantly attenuated in the insulin-treated group $(p<0.01)$, but were not reduced to the levels of control rats. The nerve glucose levels were significantly elevated in the 'Statil'-treated diabetic rats $(p<0.01)$. In the untreated diabetic rats exaggerated polyol pathway flux was indicated by the markedly elevated nerve levels of sorbitol and fructose. These were substantially reduced by 'Statil' treatment and were also attenuated in the insulintreated group, although in neither case were control dimensions achieved. Untreated diabetes was associated with a depletion of nerve myo-inositol. This was prevented by either 'Statil' or insulin treatment.

\section{Discussion}

This study has revealed marked deficits in accumulation of axonally transported phosphofructokinase activity, proximal and distal to 24 -h sciatic nerve constrictions in rats with streptozotocin-diabetes of 4 weeks' duration. It must be stressed that these experiments have revealed differences between control and diabetic rats in accumulation of enzyme activity. The activity was measured in an assay designed to generate optimal in vitro conditions. Hence it is not possible to make unequivocal extrapolations to the possible phosphofructokinase activity in vivo. For the same reason it is not possible to draw conclusions about transport or accumulation of enzyme protein per se. It is possible, on theoretical grounds at least, that a similar difference could derive from discrepancies in the level of activation of the enzyme against a background of unaltered transport of the protein itself.

The deficit in accumulation of activity may also have arisen from either reduced amount of enzyme in transit, from a reduced velocity of transport or from a combination of the two. A reduction in the amount of activity in transit would be associated, in steady state, with reduced activity per unit length nerve in the unconstricted sciatic trunk. This was indeed observed (see Table 1). The phosphofructokinase activity measured in unconstricted nerve must be an amalgam of that present in Schwann cells and that present in axons, together with a small contribution from other cell types. It is therefore not possible to extrapolate from the measured reduction in activity in the unconstricted nerves of the diabetic rats in an attempt to calculate the contribution of diminished amount of enzyme activity to the accumulation deficit. Until a method to differentiate Schwann cell from axonal activity presents itself, this will not be possible. We are left with the likelihood that a reduced amount of phosphofructokinase activity is transported in the nerves of the diabetic rats; we can make no assertions about the velocity of movement.

The reduced accumulation may be a reflection of diminished cell body output. There are indications from other work [19] that perikaryal protein synthesis may be reduced in streptozotocin-diabetic rats. It is also interesting to speculate on the relationship between the anterograde and retrograde accumulation deficits. It is possible to suggest primacy for either. It is generally agreed that retrograde transport of normal components of axoplasm (as distinct from extracellularly-derived trophic factors) represents a return to the cell body of surplus materials [20]. One might therefore suggest that diabetes could reduce a cell body output of phosphofructokinase - by mechanisms suggested elsewhere [19] - resulting in reduced anterograde transport, reduced delivery to the terminals, reduced excess and reduced return of retrogradely transported material. An alternative explanation is offered by studies on neurotoxic agents which cause peripheral axonopathies. The agents p-bromopheylacetylurea [21, 22], zinc pyridinethione [23], 2,5-hexanedione [24, 25] and acrylamide $[24,26-28]$ impair retrograde transport prior to an effect on anterograde transport. Experimental diabetes also causes an early defect of retrograde transport of glycoproteins [29]. It is therefore possible that the retrograde transport defect develops first and, as a consequence, the cell body alters the pattern of anterogradely transported components of axoplasm. Clearly the time-course of development of the defects in phosphofructokinase activity transport merit detailed study.

Both accumulation deficits were prevented completely by insulin treatment. This does not prove that hyperglycaemia was causative, but it does argue against involvement of putative neurotoxic effects of streptozotocin in the development of the defects.

Treatment of a group of diabetic rats with 'Statil' produced the amelioration of the consequences of polyol pathway flux we have come to expect from effective inhibition of aldose reductase in diabetic rats $[6-9,30]$. Thus, the failure of drug treatment to influence the phosphofructokinase activity accumulation deficits may be reconciled with firm indications of pharmacological efficacy against sciatic nerve aldose reductase. We therefore conclude that neither increased levels of sorbitol and fructose nor depletion of nerve free myo-inositol are involved in the development of the phosphofructokinase activity axonal transport defects. This is supported by our demonstration of a similar lack of effect of a different aldose reductase inhibitor [11].

The present findings provide a fascinating contrast to previous studies in which defects of axonal trans- 
port of choline acetyltransferase were found to be prevented by treatment with three different aldose reductase inhibitors $[8,9,30]$. Both choline acetyltransferase and phosphofructokinase activities move in the anterograde direction by slow transport, and one would have expected them to behave in a similar fashion in experimental diabetes. Both enzyme activities show defects of a similar magnitude but of apparently different aetiologies. It is clear from this and some previous studies $[6,7]$ that not all defects of neuronal dynamics in experimental diabetes are prevented by aldose reductase in experimental diabetes.

In the Introduction we mentioned the broader implications of studying an enzyme activity which is involved, at least in certain tissues, in the regulation of glycolysis [12]. One might have anticipated an increase in activity of phosphofructokinase activity in peripheral nerves of rats with experimental diabetes, because such animals show increased resistance to ischaemic conduction block perhaps in association with increased anaerobic ATP production [16]. However, as stated earlier in this Discussion, our finding of reduced phosphofructokinase activity - measured under optimal in vitro conditions - does not preclude increased nerve glycolysis in vivo.

\section{References}

1. Ochs S (1981) Axoplasmic transport. In: SiegelGJ, Albers RW, Agranoff BW, Katzman R (eds) Basic neurochemistry, 3rd edn. Little, Brown and Company, Boston, pp 425-442

2. Perisic M, Cuenod M (1972) Synaptic transmission depressed by colchicine blockade of axoplasmic flow. Science 175: 1140-1142

3. Volkov EM, PoletaevGI (1985) Effect of axoplasmic transport blockade on end-plate currents in frog muscle fibres. Neurophysiology 17: 140-146

4. Tomlinson DR, MayerJH (1984) Defects of axonal transport in diabetes mellitus; a possible contribution to the aetiology of diabetic neuropathy. J Autonom Pharmacol 4: 59-72

5. Sidenius P, Jakobsen J (1982) Reversibility and preventability of the decrease in slow axonal transport velocity in experimental diabetes. Diabetes 31: 689-693

6. MayerJH, Tomlinson DR, McLean WG (1984) Slow orthograde axonal transport of radiolabelled protein in sciatic motoneurones of rats with short-term experimental diabetes: effects of treatment with an aldose reductase inhibitor or myo-inositol. J Neurochem 43: 1265-1270

7. Tomlinson DR, Sidenius P, Larsen JR (1986) Slow component-a of axonal transport, nerve myo-inositol, and aldose reductase inhibition in streptozotocin-diabetic rats. Diabetes 35: 398-402

8. Mayer JH, Tomlinson DR (1983) Prevention of defects of axonal transport and nerve conduction velocity by oral administration of myo-inositol or an aldose reductase inhibitor in streptozotocindiabetic rats. Diabetologia 25: 433-438

9. Tomlinson DR, Moriarty RJ, MayerJH (1984) Prevention and reversal of defective axonal transport and motor nerve conduction velocity in rats with experimental diabetes, by treatment with the aldose reductase inhibitor Sorbinil. Diabetes 33: 470-476

10. Mayer JH, Tomlinson DR (1983) Axonal transport of cholinergic transmitter enzymes in vagus and sciatic nerves of rats with acute experimental diabetes mellitus; correlation with motor nerve conduction velocity and effects of insulin. Neuroscience 9: 951-957
11. Willars GB, Tomlinson DR, Calthrop-Owen EF (1986) Axonal transport of phosphofructokinase in experimental diabetes. Diabetic Med 3: 389 A

12. Newsholme EA (1980) Use of enzyme activity measurements in studies on the biochemistry of exercise. Int $\mathbf{J}$ Sports Med 1: 100-102

13. Steiness IB (1963) Diabetic neuropathy: vibration sense and abnormal tendon reflexes in diabetics. Acta Med Scand Suppl 394: 1-91

14. Gregersen $\mathrm{G}$ (1968) Variations in motor conduction velocity produced by acute changes of the metabolic state in diabetic patients. Diabetologia 4: 273-277

15. SeneviratneKN, Peiris OA (1969) The effects of hypoxia on the excitability of the isolated peripheral nerves of alloxan diabetic rats. J Neurol Neurosurg Psychiatry 32: 462-469

16. Low PA, Ward K, SchmelzerJD, Brimijoin S (1985) Ischaemic conduction failure and energy metabolism in experimental diabetic neuropathy. Am J Physiol 248: E457-462

17. Opie LH, Newsholme EA (1967) The activities of fructose 1,6-diphosphatase, phosphofructokinase and phosphoenolpyruvate carboxykinase in white and red muscle. Biochem J 103: 390-399

18. Edwards AL (1970) Experimental design in psychological research, 3rd edn. Holt, Rinehart and Winston, London Sydney New York Toronto, pp 130-150

19. Green RJ, King RHM, Thomas PK, Baron DN (1985) Sodium-potassium ATPase activity in the dorsal root ganglia of rats with streptozotocin-induced diabetes. Diabetologia 28: 104-107

20. Bisby MA (1980) Retrograde axonal transport. Adv Cell Neurobiol 1: $69-117$

21. Cavanagh JB, Chen FCK, Kyu MH, Ridley A (1968) The experimental neuropathy in rats caused by p-bromophenylacetylurea. $\mathbf{J}$ Neurol Neurosurg Psychiatry 31: 471-478

22. Jakobsen J, Brimijoin S (1981) Axonal transport of enzymes and labelled proteins in experimental axonopathy induced by $\mathrm{p}$ bromophenylacetylurea. Brain Res 229: 103-123

23. SahenkZ, Mendell JR (1980) Axoplasmic transport in zinc pyridine-thione neuropathy: evidence for an abnormality in distal turn-around. Brain Res 186: 343-353

24. SahenkZ, MendellJR (1981) Acrylamide and 2,5-hexanedione neuropathies: abnormal bidirectional transport rate in distal axons. Brain Res 219: 397-405

25. Braendgaard $H$, Sidenius $P$ (1986) The retrograde fast component of axonal transport in motor and sensory nerves of rat during administration of 2,5-hexanedione. Brain Res 378: 1-7

26. Souyri F, Chretien M, Droz B (1981) 'Acrylamide-induced' neuropathy and impairment of axonal transport of proteins. I. Multifocal retention of fast transported proteins at the periphery as revealed by light microscope autoradiography. Brain Res 205: 1-14

27. Jakobsen J, Sidenius P (1983) Early and dose-dependent decrease of retrograde axonal transport in acrylamide-intoxicated rats. $\mathbf{J}$ Neurochem 40: 447-454

28. Miller MS, Spencer PS (1984) Single doses of acrylamide reduce retrograde transport velocity. J Neurochem 40: 1401-1408

29. Jakobsen J, Sidenius P (1979) Decreased axonal flux of retrogradely transported glycoproteins in early experimental diabetes. J Neurochem 33: 1055-1060

30. Tomlinson DR, Townsend J, Fretten P (1985) Prevention of defective axonal transport in streptozotocin-diabetic rats by treatment with 'Statil' (ICI 128436), an aldose reductase inhibitor. Diabetes 34: 970-972

Received: 19 September 1986

and in revised form: 18 February 1987

Dr. G. B.Willars

Department of Physiology and Pharmacology

Medical School

Queen's Medical Centre

Clifton Boulevard

Nottingham NG7 2UH

UK 